\title{
Liver metastases "siphon" off immunotherapy response
}

\author{
Nina Cortese ${ }^{1}$, Federica Marchesi ${ }^{1,2}$ \\ ${ }^{1}$ Department of Immunology and Inflammation, IRCCS Humanitas Research Hospital, Milan, Italy; ${ }^{2}$ Department of Medical Biotechnology and \\ Translational Medicine, University of Milan, Milan, Italy \\ Correspondence to: Federica Marchesi. IRCCS Humanitas Research Hospital, Via Manzoni 56, 20089 Rozzano, Milan, Italy. \\ Email: federica.marchesi@humanitasresearch.it. \\ Comment on: Yu J, Green MD, Li S, et al. Liver metastasis restrains immunotherapy efficacy via macrophage-mediated T cell elimination. Nat Med \\ 2021;27:152-64.
}

Submitted May 26, 2021. Accepted for publication Jun 06, 2021.

doi: 10.21037/hbsn-21-215

View this article at: https://dx.doi.org/10.21037/hbsn-21-215

For many different cancers, the advent of immunotherapy (IT) treatments, in particular immune checkpoint inhibitors, aimed at reviving the immune response against cancer cells, has led to a great improvement in terms of clinical benefit. Despite the success of IT, even in advanced metastatic cancers, some patients still fail to respond for reasons yet unclear $(1,2)$. Yu et al. in an issue of Nature Medicine, set out to investigate whether metastatic progression to specific distant organs could systemically affect response to IT (3). The authors demonstrate that liver metastases, but not metastases to other organs, limit IT efficacy both in metastatic patients and preclinical models. The clinical significance of these findings is most relevant when considering that metastasis is the primary cancer-related cause of death, and the liver is a frequent site of metastasis $(4,5)$ and most IT are largely restricted to patients at advanced stage of disease.

Yu et al. investigated the correlation between the presence of distant metastases and response to IT in several cohorts of patients. In metastatic melanoma and nonsmall cell lung cancer (NSCLC), the authors observed a diminished response to IT in patients with metastases to the liver, as well as worse clinical outcome in terms of overall and progression free survival, independently of other clinicopathological features including PD-L1 expression and tumor burden. Due to its physiological role, the liver is constantly exposed to antigens and is therefore characterized by a tolerant immune environment, generated by immunosuppressive mechanisms such as $\mathrm{T}$ cell anergy, induction of regulatory $\mathrm{T}$ cells, and deletion of antigen-specific $\mathrm{T}$ cells (6). This could reasonably be one of the mechanisms contributing to the lack of a local responsiveness to therapy. Interestingly however, melanoma patients with liver metastases receiving IT were also shown to have increased systemic tumor burden, strongly suggesting a systemic-rather than local-effect on anti-tumor immunity. The authors further confirmed the correlation between metastatic tropism to the liver and limited therapeutic benefit from immune checkpoint blockade in a cohort of melanoma, NSCLC, urothelial and renal cancer patients, extending the relevance of these findings to a wide variety of human cancers. The tumor microenvironment has been long considered as the master regulator of antitumor immune responses, but with the advent of IT, this local view is being challenged. Cancer is a systemic disorder, perturbing the immune organization as a whole (7) and a more comprehensive picture emerges, inclusive of multiple immune cell types interacting systemically across tissues.

To unearth the mechanism underlying these variable therapeutic outcomes, the authors developed a murine model of liver metastases, where IT sensitive MC38 colorectal cancer cells were injected subcutaneously to establish the primary tumor and via intrasplenic inoculation to generate the liver metastases. Only mice harboring a primary tumor, but no concomitant liver lesions, responded to anti-PD-L1 treatment, whereas the therapeutic effect was completely abrogated in the presence of liver metastases. Results were further confirmed in a similar mouse model of lung metastases. Notably, following anti-PD-L1 treatment, primary tumors were robustly infiltrated by $\mathrm{CD} 8^{+} \mathrm{T}$ cells, which dropped in frequency in the presence of liver metastases. Naïve $T$ cell priming and activation were 

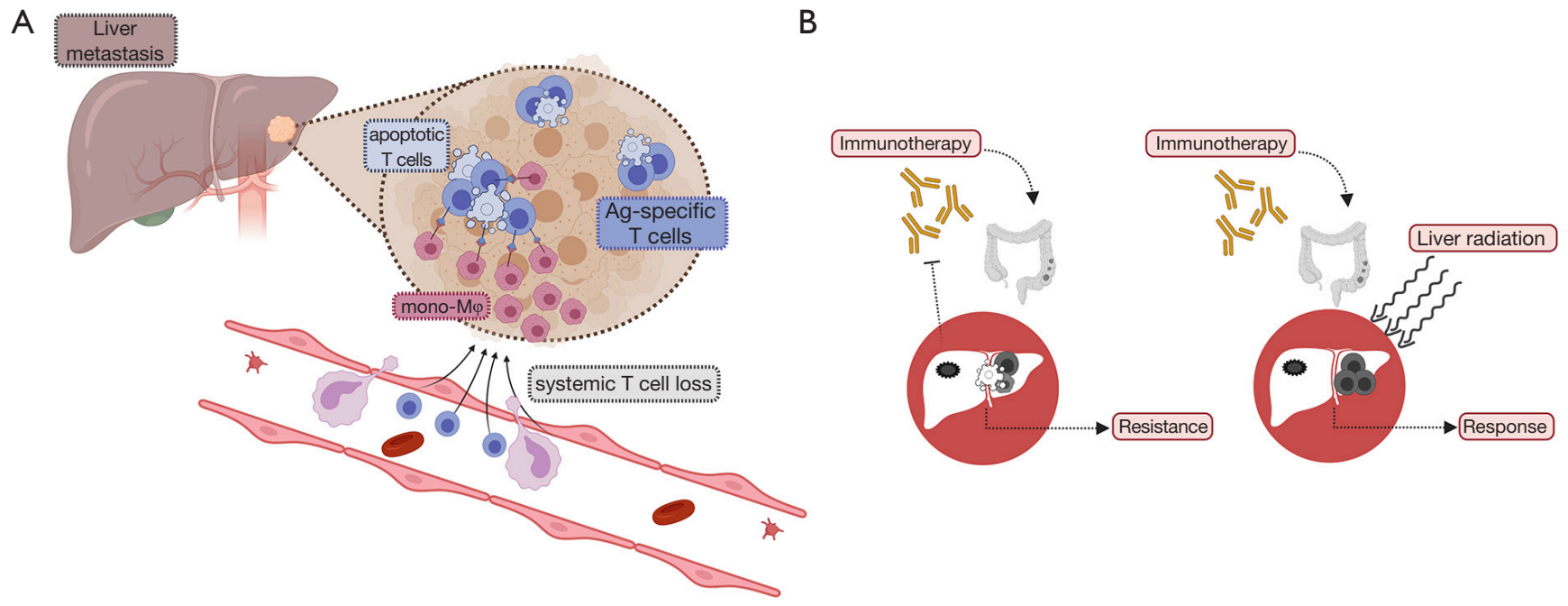

Figure 1 Macrophage-mediated T cell killing hampers systemic response to IT in liver metastases. (A) Liver metastases result in systemic $\mathrm{T}$ cell loss due to contact-dependent elimination of antigen-specific $\mathrm{T}$ cells by immunosuppressive monocyte-derived hepatic macrophages. (B) Applicability to the therapeutic setting of gastrointestinal cancers poorly responding to single-agent checkpoint inhibitors. Combination of liver radiation and IT results in response, following rewiring of the liver microenvironment. Created with BioRender.com. IT, immunotherapy.

unaffected by the presence of liver metastases, possibly suggesting that liver metastases induced a shift in antigenspecific $\mathrm{T}$ cell systemic distribution. Indeed, the numbers of antigen-specific $\mathrm{CD}^{+} \mathrm{T}$ cells were drastically reduced in primary tumors, draining lymph nodes and peripheral blood of mice harboring liver lesions. The relevance of these results to the human disease was confirmed by the mirrored finding that NCSLC patients with liver metastases had reduced absolute lymphocyte counts compared to patients without liver lesions. Comprehensive sequencing of primary tumors, including melanoma, NSCLC, breast, prostate, and colorectal cancer, from metastatic patients revealed diminished $\mathrm{T}$ cell clonality and diversity, and decreased $\mathrm{T}$ cell effector functions in patients with liver, but not lung, metastases.

To explain this systemic and liver metastases-dependent vanishing of anti-tumor $\mathrm{T}$ cells, the authors extensively evaluated the immune microenvironment of mice with hepatic lesions using flow cytometry, mass cytometry (CyTOF) and single-cell sequencing. This comprehensive approach revealed higher apoptosis and dysfunction markers in tumor antigen-specific $\mathrm{CD} 8^{+} \mathrm{T}$ cells in liver metastases compared to other sites including the primary tumor. The investigation of the hepatic immune microenvironment also highlighted an increase in monocyte-derived

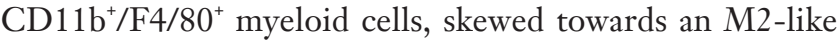
immunosuppressive phenotype by tumor cells; depletion of these cells rebalanced the numbers of apoptotic CD8 ${ }^{+}$ $\mathrm{T}$ cells in liver lesions, ultimately restoring systemic $\mathrm{T}$ cell distribution and anti-PD-L1 responsiveness. Mechanistically, hepatic macrophages expressed higher levels of FasL compared to lung macrophages, and in vitro hepatic tumor-educated macrophages induced $\mathrm{T}$ cell apoptosis in a contact dependent manner via FasL-Fas interaction (Figure 1A).

Since radiotherapy is used in the clinic for hepatic tumors and is known to stimulate anti-tumor immunity, the authors next tested the therapeutic implications of their findings utilizing liver-directed radiation, alone or in combination with anti-PD-L1, to modulate the immune infiltrate in liver metastases and improve $\mathrm{CD}^{+} \mathrm{T}$ cell cytotoxicity. Radiotherapy treatment alone was able to reduce myeloid cell frequency and increase $\mathrm{CD} 8^{+} \mathrm{T}$ cell infiltration in hepatic lesions, ascribable to a shift from myeloid to lymphocyte-recruiting cytokine production in the liver, while the combination with IT additionally led to robust regression of tumor growth and prolonged survival compared to IT alone.

The study by $\mathrm{Yu}$ et al. identifies the tolerogenic hepatic microenvironment as a key negative modulator 
of systemic IT responsiveness in metastatic patients. Specifically, the authors ascribe this phenomenon to a "siphoning" of antigen-specific CD $8^{+} \mathrm{T}$ cells to the liver, which are subsequently eliminated in a contact-dependent manner by immunosuppressive monocyte-derived hepatic macrophages. Ultimately, this leads to a systemic T cell "desert", undermining IT efficacy, which greatly relies on $\mathrm{CD}^{+} \mathrm{T}$ cell infiltration. A recent study reported a similar liver metastases-dependent effect on systemic anti-tumor immunity and IT responsiveness, however mediated by a different underlying mechanism encompassing regulatory $\mathrm{T}$ cell recruitment and activation and modulation of $\mathrm{CD}_{11 \mathrm{~b}^{+}}$ monocytes at the primary tumor site (8).

The findings elucidated by this study have widespread implications, given that most tumors metastasize to the liver. Importantly, Yu et al. also demonstrate that liverdirected radiotherapy combined with IT may be able to restore anti-tumor immunity and responsiveness to antiPDL1 treatment (Figure 1B). This approach could have a positive impact on many gastrointestinal cancers, which frequently metastasize to the liver, and currently respond poorly to single-agent checkpoint inhibitors (1), provided that long-term side effects of liver radiation are considered. Additionally, both anti-PD-L1 and radiotherapy treatments are already FDA approved for many cancer types, thereby possibly encouraging new clinical trials of combinatorial treatments.

Responsiveness to IT was restored by depleting hepatic myeloid cells, mostly macrophages, responsible for tumor antigen-specific $\mathrm{T}$ cell apoptosis. This finding further supports ongoing efforts to target macrophages, alone or in combination with checkpoint inhibitors, to limit immunosuppression behind resistance to IT (9-11). However, as also highlighted by $\mathrm{Yu} e t$ al. in this work, hepatic myeloid cells are a heterogeneous population, comprising different phenotypic and functional subsets $(12,13)$. A deeper investigation to pinpoint the macrophage subset responsible for IT refractoriness could aid in developing more effective and less toxic immunomodulatory treatments.

Collectively, this study brings to light novel mechanisms by which liver metastases hinder systemic IT efficacy and provides promising insights towards broadening immune checkpoint blockade therapeutic benefit.

\section{Acknowledgments}

Funding: None.

\section{Footnote}

Provenance and Peer Review: This article was commissioned by the editorial office of Hepatobiliary Surgery and Nutrition. The article did not undergo external peer review.

Conflicts of Interest: Both authors have completed the ICMJE uniform disclosure form (available at https://hbsn. amegroups.com/article/view/10.21037/hbsn-21-215/coif). The authors have no conflicts of interest to declare.

Ethical Statement: The authors are accountable for all aspects of the work in ensuring that questions related to the accuracy or integrity of any part of the work are appropriately investigated and resolved.

Open Access Statement: This is an Open Access article distributed in accordance with the Creative Commons Attribution-NonCommercial-NoDerivs 4.0 International License (CC BY-NC-ND 4.0), which permits the noncommercial replication and distribution of the article with the strict proviso that no changes or edits are made and the original work is properly cited (including links to both the formal publication through the relevant DOI and the license). See: https://creativecommons.org/licenses/by-nc-nd/4.0/.

\section{References}

1. Ribas A, Wolchok JD. Cancer immunotherapy using checkpoint blockade. Science 2018;359:1350-5.

2. Sharma P, Hu-Lieskovan S, Wargo JA, et al. Primary, Adaptive, and Acquired Resistance to Cancer Immunotherapy. Cell 2017;168:707-23.

3. Yu J, Green MD, Li S, et al. Liver metastasis restrains immunotherapy efficacy via macrophage-mediated $\mathrm{T}$ cell elimination. Nat Med 2021;27:152-64.

4. Ganesh K, Massagué J. Targeting metastatic cancer. Nat Med 2021;27:34-44.

5. Siegel RL, Miller KD, Jemal A. Cancer statistics, 2020. CA Cancer J Clin 2020;70:7-30.

6. Krenkel O, Tacke F. Liver macrophages in tissue homeostasis and disease. Nat Rev Immunol 2017;17:306-21.

7. Hiam-Galvez KJ, Allen BM, Spitzer MH. Systemic immunity in cancer. Nat Rev Cancer 2021;21:345-59.

8. Lee JC, Mehdizadeh S, Smith J, et al. Regulatory T cell control of systemic immunity and immunotherapy response in liver metastasis. Sci Immunol 2020;5:eaba0759. 
9. Cortese N, Donadon M, Rigamonti A, et al. Macrophages at the crossroads of anticancer strategies. Front Biosci (Landmark Ed) 2019;24:1271-83.

10. Mantovani A, Marchesi F, Malesci A, et al. Tumourassociated macrophages as treatment targets in oncology. Nat Rev Clin Oncol 2017;14:399-416.

11. Weissleder R, Pittet MJ. The expanding landscape of inflammatory cells affecting cancer therapy Nat Biomed

Cite this article as: Cortese N, Marchesi F. Liver metastases "siphon" off immunotherapy response. HepatoBiliary Surg Nutr 2021;10(4):526-529. doi: 10.21037/hbsn-21-215
Eng 2020;4:489-98.

12. Zhang Q, He Y, Luo N, et al. Landscape and Dynamics of Single Immune Cells in Hepatocellular Carcinoma. Cell 2019;179:829-45.

13. Ramachandran P, Dobie R, Wilson-Kanamori JR, et al. Resolving the fibrotic niche of human liver cirrhosis at single-cell level. Nature 2019;575:512-8. 